\title{
Cosmology with moving dark energy and the CMB quadrupole
}

\author{
José Beltrán Jiménez* and Antonio L. Maroto ${ }^{\dagger}$ \\ Departamento de Física Teórica, Universidad Complutense de Madrid, 28040 Madrid, Spain
}

(Received 29 March 2007; published 18 July 2007)

\begin{abstract}
We study the consequences of a homogeneous dark energy fluid having a nonvanishing velocity with respect to the matter and radiation large-scale rest frames. We consider homogeneous anisotropic cosmological models with four fluids (baryons, radiation, dark matter, and dark energy) whose velocities can differ from each other. Performing a perturbative calculation up to second order in the velocities, we obtain the contribution of the anisotropies generated by the fluids motion to the CMB quadrupole and compare with observations. We also consider the exact problem for arbitrary velocities and solve the corresponding equations numerically for different dark energy models. We find that models whose equation of state is initially stiffer than radiation, as for instance some tracking models, are unstable against velocity perturbations, thus spoiling the late-time predictions for the energy densities. In the case of scaling models, the contributions to the quadrupole can be non-negligible for a wide range of initial conditions. We also consider fluids moving at the speed of light (null fluids) with positive energy and show that, without assuming any particular equation of state, they generically act as a cosmological constant at late times. We find the parameter region for which the models considered could be compatible with the measured (low) quadrupole.
\end{abstract}

DOI: 10.1103/PhysRevD.76.023003

PACS numbers: 95.36.+x, 98.80.- $\mathrm{k}$

\section{INTRODUCTION}

The present observational evidence from supernovae type Ia [1,2], CMB anisotropies [3], and large-scale structure, mainly through the baryon acoustic oscillations, suggests that today the Universe could be dominated by a negative pressure fluid [4,5]. Although these data are compatible with the presence of a cosmological constant, the fact that such observations only explore relatively recent epochs implies that other possible models in which the equation of state of dark energy could have changed in time cannot be excluded a priori. Thus, if dark energy can be parametrized as a perfect fluid with equation of state $p_{\mathrm{DE}}=w_{\mathrm{DE}}(z) \rho_{\mathrm{DE}}$, the above mentioned observations only constrain the present value as $w_{\mathrm{DE}}^{0}=-0.97_{-0.09}^{+0.07}$ [3]. The redshift dependence of the equation of state can be parametrized in different ways and still a wide range of variability is compatible with observations [6]. Moreover, apart from including new components in the energymomentum tensor within Einstein gravity, or new scalar fields as in the quintessence models [7], it has been also suggested that the accelerated expansion of the Universe could be due to modifications of the gravitational action at large distances [8].

The possibility of finding observational signals of dark energy which could discriminate between the various models is thus becoming of crucial importance. However, up to date, only a few proposals have been considered in the literature, which can be broadly classified in two classes [9]: on one hand probes of the redshift-distance relation, as for instance the already mentioned high-redshift superno-

\footnotetext{
*jobeltra@fis.ucm.es

maroto@fis.ucm.es
}

vae Ia or the use of baryon acoustic oscillations as standard rulers. On the other hand we have the probes of the growth of structure in the Universe, such as the weak gravitational lensing or the integrated Sachs-Wolfe effect. The goals of the future observational surveys will be the determination of the equation of state of dark energy with a few percent accuracy and the possibility of discriminating from a pure cosmological constant [10].

In this paper we consider a different aspect of dark energy with potential observational consequences which is related to the possible motion of dark energy with respect to matter and radiation. If dark energy can be described as a homogeneous perfect fluid then, apart from the density parameter $\Omega_{\mathrm{DE}}$ and equation of state $w_{\mathrm{DE}}$, a complete knowledge of its energy-momentum tensor requires the determination of its relative velocity with respect to the rest of the components of the Universe. Indeed, dark energy is usually considered as a highly homogeneous fluid in a similar way to radiation. This is due to the fact that in most models, its sound speed is close to the speed of light and this prevents the growth of dark energy perturbations below the Hubble scale. Moreover, dark energy is also required to be extremely weakly interacting with baryons and radiation, in order to avoid conflicts with the predictions of standard cosmology. Indeed, in most of the models, its effects are purely gravitational and dark energy is considered as a totally decoupled fluid. In such a case it makes sense to ask whether the dark energy rest frame converges towards the radiation or matter large-scale rest frames. Since there is no a priori reason to expect that dark energy were necessarily coupled to radiation in the very early Universe, its initial velocity with respect to radiation should be considered as a free cosmological parameter on equal footing to $\Omega_{\mathrm{DE}}$ or $w_{\mathrm{DE}}$, and very 
much in the same way as those parameters, the relative velocity of dark energy should be determined by observations. In addition, the fact that a pure cosmological constant is invariant under change of frame implies that the potential effects associated to a nonvanishing relative velocity will be exclusively present in models with varying equation of state.

The metric anisotropies generated by the fluids motion can affect both the temperature and polarization of the CMB. In a previous work [11], we have started the study of such effects on the CMB dipole. We have shown that the motion of dark energy is not incompatible with the current measurements of the dipole, but instead, it modifies its usual interpretation. Thus, when dark energy is moving, the dipole is generated by the motion of emitter and observer with respect to the cosmic center of mass and not with respect to the background radiation. This fact can also have important consequences for the generation of matter bulk flows on very large scales [12]. In this work we consider the effects of motion on the CMB quadrupole. In the last years, the interest in anisotropic models (see $[13,14]$ and references therein) has grown mainly motivated by some unexpected features in the low multipoles of the CMB temperature anisotropies, in particular, the low value of the quadrupole and the quadrupole-octupole alignment which could suggest the existence of a preferred direction in the Universe $[15,16]$. In this work we explore this possibility by considering cosmologies with several moving fluids (see [17] for previous works).

The paper is organized as follows: in Sec. II we obtain the metric solution for a model with moving fluids up to second order in the velocities. In Sec. III, we obtain the corresponding CMB temperature anisotropies and the contribution to the quadrupole. In Sec. IV we consider the exact problem for large velocities and obtain the general expression for the quadrupole. Section V is devoted to the application of the previous results to different dark energy models. Finally Sec. VI contains the main conclusions of the paper.

\section{SLOW-MOVING FLUIDS: SECOND ORDER EQUATIONS}

Let us consider a universe filled with four homogeneous perfect fluids: baryons, radiation, dark matter, and dark energy. For matter and radiation we shall consider their usual equations of state, i.e., $p_{R}=\frac{1}{3} \rho_{R}$ and $p_{B}=p_{\mathrm{DM}}=$ 0 , whereas for dark energy we shall use: $p_{\mathrm{DE}}=$ $w_{\mathrm{DE}}(z) \rho_{\mathrm{DE}}$, which in general depends on redshift. In an arbitrary frame, the energy-momentum tensor for each component takes the perfect fluid form

$$
T_{\alpha}^{\mu \nu}=\left(\rho_{\alpha}+p_{\alpha}\right) u_{\alpha}^{\mu} u_{\alpha}^{\nu}-p_{\alpha} g^{\mu \nu},
$$

where $\alpha=B, R$, DM, DE. We shall consider homogeneity in the fluids so that all the quantities appearing in (1) will just depend on conformal time

$$
\rho_{\alpha}=\rho_{\alpha}(\eta), \quad u_{\alpha}^{\mu}=\gamma_{\alpha}(\eta)\left(1, \vec{v}_{\alpha}(\eta)\right)
$$

with

$$
\gamma_{\alpha}=\frac{1}{\sqrt{g_{00}+g_{i j} v_{\alpha}^{i} v_{\alpha}^{j}}} .
$$

In order to simplify the problem and to obtain some analytical solutions we shall use perturbation theory, assuming that the fluids velocities are small, i.e. $\vec{v}_{\alpha}^{2} \ll 1$. To that end, we expand the different quantities of the four fluids up to second order as follows:

$$
\begin{aligned}
& \rho_{\alpha}=\rho_{\alpha}^{(0)}+\rho_{\alpha}^{(1)}+\rho_{\alpha}^{(2)}+\cdots, \\
& \vec{v}_{\alpha}=\vec{v}_{\alpha}^{(1)}+\vec{v}_{\alpha}^{(2)}+\cdots,
\end{aligned}
$$

where we have imposed the fluids to be at rest to zeroth order, i.e., $\vec{v}_{\alpha}^{(0)}=0$. That way, the most general form for the metric is given by the perturbed Friedmann-RobertsonWalker metric:

$$
\begin{aligned}
d s^{2}= & a^{2}\left[\left[1+2\left(\phi^{(1)}+\phi^{(2)}\right)\right] d \eta^{2}+2\left[S_{i}^{(1)}+S_{i}^{(2)}\right] d x^{i} d \eta\right. \\
& \left.-\left[\left(1-2\left(\psi^{(1)}+\psi^{(2)}\right)\right) \delta_{i j}+h_{i j}\right] d x^{i} d x^{j}\right],
\end{aligned}
$$

where we will follow the notation in [18]. In this expression, $\phi^{(1)}$ and $\psi^{(1)}$ are scalar perturbations of first order and will be determined from $\rho_{\alpha}^{(1)}$ in the first order equations of motion. However, the second order scalar perturbations $\phi^{(2)}$ and $\psi^{(2)}$ can depend, not only on $\rho_{\alpha}^{(2)}$ and $\left(\rho_{\alpha}^{(1)}\right)^{2}$ terms, but also on $\left(\vec{v}_{\alpha}^{(1)}\right)^{2}$ which are also scalars. Analogously, the first order vector perturbations $\vec{S}^{(1)}$ can only be related to the first order velocities $\vec{v}_{\alpha}^{(1)}$ in the equations of motion, whereas to second order, $\vec{S}^{(2)}$ will be determined by combinations of $\overrightarrow{\boldsymbol{v}}_{\alpha}^{(2)}$ and $F^{(1)} \overrightarrow{\boldsymbol{v}}_{\alpha}^{(1)}$ where $F^{(1)}$ is a scalar function of the first order scalar perturbations. Finally, $h_{i j}$ is a traceless tensor perturbation which should be of second order and depend on the combinations $v_{\alpha i}^{(1)} v_{\alpha j}^{(1)}-\frac{1}{3} \times$ $\left(v_{\alpha}^{(1)}\right)^{2} \delta_{i j}$. Note that, since we are considering only timedependent perturbations on the energy-momentum tensor, all the perturbations on the metric will be functions only of time and, therefore, the perturbed metric does not contain any terms involving spatial derivatives.

So far, we have not done any specific gauge choice, so we still have four gauge degrees of freedom. Hence, we can simplify the problem by choosing our coordinates appropriately. In particular, we can fix the spatial coordinates so that the vector part of the metric vanishes $\vec{S}=0$. The physical interpretation of this condition is apparent when solving the $(0 i)$ Einstein equation of the exact problem. Thus, we obtain the condition

$$
S_{i}=\frac{\sum_{\alpha} \gamma_{\alpha}^{2}\left(\rho_{\alpha}+p_{\alpha}\right) g_{i j} v_{\alpha}^{j}}{\sum_{\alpha} \gamma_{\alpha}^{2}\left(\rho_{\alpha}+p_{\alpha}\right)},
$$


where the metric is $d s^{2}=g_{\mu \nu} d x^{\mu} d x^{\nu}$. Hence, $\vec{S}$ can be interpreted as the relativistic cosmic center of mass velocity (see [11]). Notice that in general, an observer at rest with respect to cosmic center of mass could be moving with respect to radiation or matter. On the other hand, the temporal coordinate can be chosen in such a way that $\sum_{\alpha}\left(\rho_{\alpha}-\rho_{\alpha}^{(0)}\right)=0$, which means that the total density perturbations are identically zero. With this gauge choice, the $(00)$ and $(i j)$ components of Einstein equations $G^{\mu}{ }_{\nu}=$ $8 \pi G T^{\mu}{ }_{\nu}$ up to second order adopt the form:

Zeroth order

$$
\mathcal{H}^{2}=\frac{8 \pi G}{3} a^{2} \sum_{\alpha} \rho_{\alpha}^{(0)},
$$

$$
2 \mathcal{H}^{\prime}+\mathcal{H}^{2}=-8 \pi G a^{2} \sum_{\alpha} p_{\alpha}^{(0)} .
$$

First order

$$
-\frac{6}{a^{2}} \mathcal{H}\left(\psi^{\prime(1)}+\mathcal{H} \phi^{(1)}\right)=0,
$$

$$
\psi^{\prime \prime(1)}+2 \mathcal{H} \psi^{\prime(1)}+\mathcal{H} \phi^{\prime(1)}+\left(\mathcal{H}^{2}+2 \mathcal{H}^{\prime}\right) \phi^{(1)}=0 .
$$

Second order

$-2 \mathcal{H}\left(\psi^{(2)}+\left(\psi^{(1)}\right)^{2}\right)^{\prime}-2 \mathcal{H}^{2}\left(\phi^{(2)}-2\left(\phi^{(1)}\right)^{2}\right)+\psi^{\prime(1)}\left(\psi^{\prime(1)}+4 \mathcal{H} \phi^{(1)}\right)=\frac{8 \pi G}{3} a^{2} \sum_{\alpha}\left(\rho_{\alpha}^{(0)}+p_{\alpha}^{(0)}\right)\left(v_{\alpha}^{(1)}\right)^{2}$,

$$
\begin{aligned}
& \frac{2}{a^{2}}\left[\left(2 \mathcal{H}^{\prime}+\mathcal{H}^{2}\right) \phi^{(2)}+\mathcal{H} \phi^{\prime(2)}+\psi^{\prime \prime(2)}+2 \mathcal{H} \psi^{\prime(2)}-2\left(\mathcal{H}^{2}+2 \mathcal{H}^{\prime}\right)\left(\phi^{(1)}\right)^{2}+\frac{1}{2} \psi^{\prime(1)}\left(\psi^{(1)}-2 \phi^{(1)}\right)^{\prime}\right. \\
& \left.\quad+2 \psi^{\prime \prime(1)}\left(\psi^{(1)}-\phi^{(1)}\right)+2 \mathcal{H}\left[\left(\left(\psi^{(1)}\right)^{2}+\left(\phi^{(1)}\right)^{2}\right)^{\prime}+2 \phi^{(1)} \psi^{\prime(1)}\right]\right] \delta_{j}^{i}+\frac{1}{2 a^{4}}\left(a^{2} h_{j}^{\prime i}\right)^{\prime}=8 \pi G \sum_{\alpha}\left(\rho_{\alpha}^{(0)}+p_{\alpha}^{(0)}\right) v_{\alpha}^{(1) i} v_{\alpha j}^{(1)}
\end{aligned}
$$

with $^{\prime} \equiv \frac{d}{d \eta}$ and $\mathcal{H}=a^{\prime} / a$ is the Hubble parameter.

Nevertheless, the system is incomplete because there are more unknown variables than equations. In general, the problem with $n$ fluids has ten independent Einstein equations, but the unknown quantities are the densities (assuming a given equation of state) and the three independent components of the four-velocity of each fluid (because of the constraint $u^{2}=1$ ). Therefore, there are $10+4 n$ unknown functions, although, since there are four gauge degrees of freedom, we can fix four quantities and reduce the number of undetermined functions to $6+4 n$. With this count, one needs $4(n-1)$ additional equations to complete the system. The simplest way to close the problem is by requiring the conservation of each energy-momentum tensor, assuming they are decoupled from each other: $T_{\alpha}^{\mu \nu}=$ 0 . Obviously, one can modify these relations by changing the right-hand side in order to consider interactions between the fluids. This guarantees the completeness of the system since it provides the $4(n-1)$ required equations (there are $4 n$ extra equations, but the conservation of the total energy-momentum tensor makes one of those equations superfluous). For our case, these additional equations read for the energy and momentum conservation (notice that momentum conservation is trivial at zeroth order):

Zeroth order

$$
\rho_{\alpha}^{\prime(0)}+3 \mathcal{H}\left(\rho_{\alpha}^{(0)}+p_{\alpha}^{(0)}\right)=0
$$

\section{First order}

$$
\begin{gathered}
\rho_{\alpha}^{\prime(1)}+3 \mathcal{H}\left(\rho_{\alpha}^{(1)}+p_{\alpha}^{(1)}\right)=3\left(\rho_{\alpha}^{(0)}+p_{\alpha}^{(0)}\right) \psi^{\prime(1)}, \\
{\left[a^{4}\left(\rho_{\alpha}^{(0)}+p_{\alpha}^{(0)}\right) \vec{v}_{\alpha}^{(1)}\right]^{\prime}=0 .}
\end{gathered}
$$

Second order

$$
\begin{aligned}
& \rho_{\alpha}^{\prime(2)}+3 \mathcal{H}\left(\rho_{\alpha}^{(2)}+p_{\alpha}^{(2)}\right) \\
& =-\frac{1}{a^{4}}\left[a^{4}\left(\rho_{\alpha}^{(0)}+p_{\alpha}^{(0)}\right)\right]^{\prime}\left(\overrightarrow{\boldsymbol{v}}_{\alpha}^{(1)}\right)^{2}-\left(\rho_{\alpha}^{(0)}+p_{\alpha}^{(0)}\right)\left(\left(\overrightarrow{\boldsymbol{v}}_{\alpha}^{(1)}\right)^{2}\right)^{\prime} \\
& \quad+3\left[\left(\rho_{\alpha}^{(0)}+p_{\alpha}^{(0)}\right)\left(\left(\psi^{(1)}\right)^{2}+\psi^{(2)}\right)^{\prime}+\left(\rho_{\alpha}^{(1)}+p_{\alpha}^{(1)}\right) \psi^{\prime(1)}\right],
\end{aligned}
$$

$$
\begin{aligned}
& {\left[a^{4}\left(\left(\rho_{\alpha}^{(0)}+p_{\alpha}^{(0)}\right)\left(\overrightarrow{\boldsymbol{v}}_{\alpha}^{(2)}-2 \phi^{(1)} \overrightarrow{\boldsymbol{v}}_{\alpha}^{(1)}\right)+\left(\rho_{\alpha}^{(1)}+p_{\alpha}^{(1)}\right) \overrightarrow{\boldsymbol{v}}_{\alpha}^{(1)}\right)\right]^{\prime}} \\
& \quad=a^{4}\left(\rho_{\alpha}^{(0)}+p_{\alpha}^{(0)}\right)\left(5 \psi^{(1)}-\phi^{(1)}\right)^{\prime} \overrightarrow{\boldsymbol{v}}_{\alpha}^{(1)}
\end{aligned}
$$

where we have used the previous orders equations at each order.

\section{CONTRIBUTIONS TO THE CMB QUADRUPOLE}

The relevant part of the metric for the quadrupole is given in our case just by $h_{i j}$, since it is the only part contributing to the anisotropy. The homogeneous scalar perturbations only affect the value of the monopole in a 
negligible way. Concerning vector perturbations, we are working in the cosmic center of mass frame and accordingly those contributions vanish in our calculations. As commented in the introduction, the $\vec{S}$ contributions have been studied in [11] and modify the usual dipole contribution. Therefore, in order to calculate the quadrupole produced by this metric, we can consider just the tensor perturbation. Then, from now on, we shall use the metric

$$
d s^{2}=a^{2}\left(d \eta^{2}-\left(\delta_{i j}+h_{i j}\right) d x^{i} d x^{j}\right) .
$$

In order to calculate all the contributions to the temperature anisotropies generated by the metric perturbations, we should solve the corresponding radiative transfer equations (see $[19,20]$ ). This is the system of Einstein-Boltzmann equations for the set of fluids. However, since we are only interested in the quadrupole anisotropy (which is not affected by microphysics at the time of recombination), the only relevant contribution for such a large angle contribution would be given by the Sachs-Wolfe effect which takes into account the variation in the energy of photons propagating from the last scattering surface [20]

$$
\frac{\delta T}{T}=\frac{a_{0} \mathcal{E}_{0}-a_{\mathrm{dec}} \mathcal{E}_{\mathrm{dec}}}{a_{\mathrm{dec}} \mathcal{E}_{\mathrm{dec}}} .
$$

Here, the indices 0 and dec denote the present and decoupling times, respectively, and $\mathcal{E}$ is the energy of the photon. For an observer with velocity $u^{\mu}=\gamma(1, \vec{v})$ this energy is given by

$$
\mathcal{E}=g_{\mu \nu} u^{\mu} P^{\nu}
$$

with

$$
P^{\nu}=E \frac{d x^{\nu}}{d \lambda},
$$

where $E$ parametrizes the photon energy and $\lambda$ is an affine parameter. By the invariance of the action of the geodesics of a massless particle under conformal transformations of the affine parameter, the geodesics of the metric $g_{\mu \nu}$ given by (18) with affine parameter $\lambda$ are the same as those of the metric $\hat{g}_{\mu \nu}=a^{-2} g_{\mu \nu}$ with affine parameter $\eta$ such that $d \lambda=a^{2} d \eta$. The trajectory of the photon coming from the direction given by the Minkowski-null vector $n^{\mu}=(1, \vec{n})$ with $\vec{n}^{2}=1$ will be perturbed in such a way that we can write $x^{\mu}(\eta)=n^{\mu} \eta+\delta x^{\mu}$, where the second term corresponds to the contribution from $h_{i j}$ which is of second order. Then, assuming that the observer velocity is of first order, the momentum of the photon to second order is

$$
P^{\nu}=\frac{E}{a^{2}}\left(n^{\nu}+\frac{d \delta x^{\nu}}{d \eta}\right) .
$$

Inserting this expression in (20) we obtain

$$
\mathcal{E}=\frac{E}{a}\left(1+\frac{1}{2} \vec{v}^{2}-\vec{v} \cdot \vec{n}+\frac{d \delta x^{0}}{d \eta}\right) .
$$

For the $\hat{g}_{\mu \nu}$ metric, the second order of the zero component of the geodesic equations in terms of the metric perturbation reduces to

$$
\frac{d^{2} \delta x^{0}}{d \eta^{2}}+\frac{1}{2} \frac{d h_{i j}}{d \eta} n^{i} n^{j}=0
$$

which can be easily integrated to get

$$
\frac{d \delta x^{0}}{d \eta}=-\frac{1}{2} h_{i j} n^{i} n^{j}
$$

Then, the energy of the photon results finally

$$
\mathcal{E}=\frac{E}{a}\left(1+\frac{1}{2} \vec{v}^{2}-\vec{v} \cdot \vec{n}-\frac{1}{2} h_{i j} n^{i} n^{j}\right) .
$$

Thus, by using this formula for the energy of the photon in Eq. (19) and expanding up to second order we obtain the following expression for the temperature fluctuations:

$$
\begin{aligned}
\frac{\delta T}{T} \simeq & \left.\frac{1}{2} \vec{v}^{2}\right|_{\mathrm{dec}} ^{0}-\left.\vec{v} \cdot \vec{n}\right|_{\mathrm{dec}} ^{0}-\left.\left(\vec{v}_{\mathrm{dec}} \cdot \vec{n}\right)(\vec{v} \cdot \vec{n})\right|_{\mathrm{dec}} ^{0} \\
& -\left.\frac{1}{2} h_{i j} n^{i} n^{j}\right|_{\mathrm{dec}} ^{0} .
\end{aligned}
$$

The first term in (27) only contributes to the monopole and the second term is a Doppler effect, although notice that since the velocities appearing in $\left.\vec{v} \cdot \vec{n}\right|_{\mathrm{dec}} ^{0}$ are referred to the $\vec{S}=0$ frame, in the case of moving fluids, the dipole is due to the motion of emitter and observer with respect to the cosmic center of mass, as commented above. Finally, the last two terms do contribute to the quadrupole. The first of them does not depend on the metric perturbation since it comes from the second order expansion of the denominator in (19). As we will show below, this term is expected to be smaller than the last one. Therefore, the dominant contribution for the quadrupole is given by the following expression:

$$
\frac{\delta T_{Q}}{T}=-\frac{1}{2}\left(h_{i j}\left(a_{0}\right)-h_{i j}\left(a_{\mathrm{dec}}\right)\right) n^{i} n^{j} .
$$

This formula shows that we only need to know $h_{i j}$ in order to calculate the quadrupole and, besides, this term does not depend on the observer velocity. Moreover, it is easy to see from (12) that the solution for $h_{i j}$ is the following:

$$
\begin{aligned}
h_{i j}= & 6 \int_{a_{*}}^{a} \frac{1}{\tilde{a}^{4}}\left[\int_{a_{*}}^{\tilde{a}} \hat{a}^{2} \sum_{\alpha}\left(\rho_{\alpha}^{(0)}+p_{\alpha}^{(0)}\right)\left(v_{\alpha i}^{(1)} v_{\alpha j}^{(1)}-\frac{1}{3} \overrightarrow{\boldsymbol{v}}_{\alpha}^{(1) 2} \delta_{i j}\right)\right. \\
& \left.\times \frac{d \hat{a}}{\sqrt{\sum_{\alpha} \rho_{\alpha}^{(0)}}}\right] \frac{d \tilde{a}}{\sqrt{\sum_{\alpha} \rho_{\alpha}^{(0)}}},
\end{aligned}
$$

where $a_{*}$ is the value of the scale factor at the time at which we specify the initial conditions for $h_{i j}$. Notice that the quadrupole does not depend on $h_{i j}\left(a_{*}\right)$, but only on the initial value of the derivatives. For simplicity we will assume that the metric anisotropies are generated by the 
fluids motion and therefore we consider a purely isotropic universe for $a<a_{*}$, i.e., we take $h_{i j}\left(a_{*}\right)=h_{i j}^{\prime}\left(a_{*}\right)=0$.

As we can see in the last expression, the quadrupole depends on both the zeroth order densities of the fluids and the first order of the velocities with respect to the center of mass. These quantities can be obtained from the conservation equations (13) and (15). The zeroth order equations have the well-known solutions for the densities $\rho_{\alpha}^{(0)}=$ $\rho_{0 \alpha} a^{-3\left(w_{\alpha}+1\right)}, \rho_{0 \alpha}$ being the densities today. On the other hand, this expression for the zeroth order densities allows us to obtain the solutions for the velocities

$$
\vec{v}_{\alpha}^{(1)}=\vec{v}_{0 \alpha} a^{3 w_{\alpha}-1},
$$

where $\overrightarrow{\boldsymbol{v}}_{0 \alpha}$ are the velocities of the fluids today. We see that, to first order, each fluid will move along a fixed direction. Well inside the radiation-dominated era, baryons and dark matter particles were coupled to radiation which, being the dominant component, will drag matter in such a way that the three fluids velocities are the same. From decoupling on, each fluid velocity will start evolving according to (30), i.e. matter will start reducing its velocity with respect to the cosmic center of mass frame, whereas radiation keeps moving at a constant velocity. Since dark matter is expected to decouple before baryons do, and both velocities scale in the same way, the two matter fluids are expected to be moving with constant relative velocity after recombination. Finally since the initial velocity of the three fluids (radiation, baryons, and dark matter) were the same, the direction of their velocities will also agree after recombination. On the other hand, the gauge condition $\vec{S}=0$ in (6) yields the constraint

$$
\sum_{\alpha}\left(\rho_{\alpha}^{(0)}+p_{\alpha}^{(0)}\right) \overrightarrow{\boldsymbol{v}}_{\alpha}^{(1)}=0
$$

so we can conclude that dark energy should also move in the same direction as the rest of fluids in this frame. That way, each energy-momentum tensor (and therefore the total one) will have axisymmetry so the metric will also be axisymmetric. This means that the tensor perturbation $h_{i j}$ is diagonal. In fact, if we choose the velocities along the $z$-axis, the tensor perturbation given by (29) will be proportional to $\operatorname{diag}(-1,-1,2)$. Taking into account the previous discussion, the final expression for the quadrupole results:

$$
\frac{\delta T_{Q}}{T}=-\frac{1}{2}\left(h\left(a_{0}\right)-h\left(a_{\mathrm{dec}}\right)\right)\left(\cos ^{2} \theta-\frac{1}{3}\right),
$$

where $\theta$ is the angle formed by the observation direction and the velocities of the fluids, and $h(a)=\sum_{\alpha} h_{\alpha}(a)$ with

$$
\begin{aligned}
h_{\alpha}(a)= & 6 \int_{a_{*}}^{a} \frac{1}{\tilde{a}^{4}}\left[\int_{a_{*}}^{\tilde{a}} \hat{a}^{2}\left(\rho_{\alpha}^{(0)}+p_{\alpha}^{(0)}\right) v_{\alpha}^{(1) 2} \frac{d \hat{a}}{\sqrt{\sum_{\alpha} \rho_{\alpha}^{(0)}}}\right] \\
& \times \frac{d \tilde{a}}{\sqrt{\sum_{\alpha} \rho_{\alpha}^{(0)}}} .
\end{aligned}
$$

The function of $\theta$ appearing in (32) is proportional to the spherical harmonic $Y_{20}$ so we can express the quadrupole fluctuation as

$$
\frac{\delta T_{Q}}{T}=\frac{2}{3} \sqrt{\frac{\pi}{5}}\left(h_{0}-h_{\mathrm{dec}}\right) Y_{20} .
$$

It is usual to introduce the power spectrum of the temperature fluctuations of $\mathrm{CMB}$ as

$$
\frac{\delta T_{\ell}}{T}=\sqrt{\frac{1}{2 \pi} \frac{\ell(\ell+1)}{2 \ell+1} \sum_{m}\left|a_{\ell m}\right|^{2}},
$$

where $a_{\ell m}$ are the coefficients of the expansion in spherical harmonics. Moreover, the quadrupole is usually defined as

$$
Q \equiv \frac{\delta T_{2}}{T}=\sqrt{\frac{3}{5 \pi} \sum_{m=-2}^{2}\left|a_{2 m}\right|^{2}}
$$

which, in our case, reduces to

$$
Q_{A}=\frac{2}{5 \sqrt{3}}\left|h_{0}-h_{\mathrm{dec}}\right| .
$$

The quadrupole given by (37) is due to the anisotropy of the space-time background (that is why the index $A$ is introduced), but we have to add the standard isotropic fluctuation produced during inflation. Then, if we assume that the anisotropies are small, the total effect will be the linear superposition of both contributions (see [14]):

$$
\delta T_{T}=\delta T_{A}+\delta T_{I}
$$

and, therefore

$$
a_{\ell m}^{T}=a_{\ell m}^{A}+a_{\ell m}^{I} .
$$

Notice that, as discussed in [14], there is the possibility that the inflation-produced contribution could be strongly biased or antibiased by the anisotropic background, mainly in the case in which anisotropies grew as we go back in time. However as discussed in that reference, this is unlikely in general since it would require a correlation between the quantum origin and subsequent classical evolution. Moreover, in our case, the background evolution during inflation is isotropic and we do not expect any interference effect.

Following [21], we can easily generalize our results to the case of an arbitrary orientation of our frame in which the velocities lie along the direction given by $(\hat{\theta}, \hat{\phi})$. In that case, the coefficients of the expansion are 


$$
\begin{aligned}
& a_{20}^{A}=\frac{\sqrt{\pi}}{6 \sqrt{5}}[1+3 \cos 2 \hat{\theta}]\left|h_{0}-h_{\mathrm{dec}}\right|, \\
& a_{21}^{A}=-\left(a_{2-1}^{A}\right)^{*}=-\sqrt{\frac{\pi}{30}} e^{-i \hat{\phi}} \sin 2 \hat{\theta}\left|h_{0}-h_{\mathrm{dec}}\right|, \\
& a_{22}^{A}=\left(a_{2-2}^{A}\right)^{*}=\sqrt{\frac{\pi}{30}} e^{-2 i \hat{\phi}} \sin ^{2} \hat{\theta}\left|h_{0}-h_{\mathrm{dec}}\right| .
\end{aligned}
$$

It is easy to show that the anisotropy quadrupole according to (36) is still given by (37) since $h$ is scalar under rotations. Now, assuming that the coefficients $a_{2 m}^{I}$ only differ one from each other in a phase factor we can write

$$
\begin{aligned}
& a_{20}^{I}=\sqrt{\frac{\pi}{3}} e^{i \alpha_{1}} Q_{I}, \\
& a_{21}^{I}=-\left(a_{2-1}^{I}\right)^{*}=\sqrt{\frac{\pi}{3}} e^{i \alpha_{2}} Q_{I}, \\
& a_{22}^{I}=\left(a_{2-2}^{I}\right)^{*}=\sqrt{\frac{\pi}{3}} e^{i \alpha_{3}} Q_{I}
\end{aligned}
$$

which is justified because the standard inflation fluctuations are statistically isotropic. Then, the total quadrupole can be expressed as

$$
Q_{T}^{2}=Q_{A}^{2}+Q_{I}^{2}-2 f Q_{A} Q_{I}
$$

where $f$ is a function depending on the direction of the velocities $(\hat{\theta}, \hat{\phi})$ and the phase factors $\alpha_{i}$ of the coefficients $a_{2 m}^{I}$, and whose expression is

$$
\begin{aligned}
f= & \frac{1}{4 \sqrt{5}}\left[2 \sqrt{6}\left[-\sin \hat{\theta} \cos \left(2 \hat{\phi}+\alpha_{3}\right)+2 \cos \hat{\theta} \cos \left(\hat{\phi}+\alpha_{2}\right)\right]\right. \\
& \left.\times \sin \hat{\theta}-(1+3 \cos (2 \hat{\theta})) \cos \alpha_{1}\right] .
\end{aligned}
$$

This function takes values such that

$$
|f| \leq \bar{f}=\frac{\sqrt{39+6 \sqrt{6}}+\sqrt{6}-1}{4 \sqrt{5}} .
$$

Since the values of the phases $\alpha_{i}$ are random, the total quadrupole lies between $Q_{+}^{2}$ and $Q_{-}^{2}$, being

$$
Q_{ \pm}^{2}=Q_{A}^{2}+Q_{I}^{2} \pm 2 \bar{f} Q_{A} Q_{I}
$$

with $\bar{f}$ the maximum of $f$.

The observed quadrupole from WMAP [3] is given by $(\delta T)_{\mathrm{obs}}^{2}=236_{-137}^{+560} \mu \mathrm{K}^{2}$ at the $68 \%$ C.L. or $(\delta T)_{\mathrm{obs}}^{2}=$ $236_{-182}^{+3591} \mu \mathrm{K}^{2}$ at the $95 \%$ C.L. These results define the corresponding $68 \%$ C.L or $95 \%$ C.L. intervals for the measured temperature fluctuations that we denote: $\left((\delta T)_{\min }^{2},(\delta T)_{\max }^{2}\right)$. For the theoretical quadrupole temperature interval $\left((\delta T)_{-}^{2},(\delta T)_{+}^{2}\right)$, obtained from (45), to be compatible with observations, we therefore require $(\delta T)_{\max }^{2} \geq(\delta T)_{-}^{2}$ and $(\delta T)_{\min }^{2} \lesssim(\delta T)_{+}^{2}$. Using (45) these two conditions impose limits on $\left(\delta T_{A}\right)^{2}$ once the value of $(\delta T)_{I}^{2}$ is fixed.

Let us first assume that inflation alone is able to account for the observed quadrupole, i.e., $(\delta T)_{I}^{2} \simeq 236 \mu \mathrm{K}^{2}$, then the first condition $(\delta T)_{\max }^{2} \geq(\delta T)_{-}^{2}$ is automatically satisfied, because the minimum of $(\delta T)_{+}^{2}$, as a function of $\delta T_{A}$, is $(\delta T)_{I}^{2}$ which is larger than $(\delta T)_{\min }^{2}$. Therefore, we obtain bounds on $\delta T_{A}$ just from the second condition above, which are given by

$$
\begin{array}{ll}
0 \mu \mathrm{K}^{2} \lesssim\left(\delta T_{A}\right)^{2} \lesssim 1861 \mu \mathrm{K}^{2} \quad 68 \% \text { C.L. }, \\
0 \mu \mathrm{K}^{2} \lesssim\left(\delta T_{A}\right)^{2} \lesssim 5909 \mu \mathrm{K}^{2} \quad \text { 95\%C.L. }
\end{array}
$$

However, it is well known that the predictions of standard inflation, calculated from an almost flat spectrum of density perturbations, is larger than the central value of the measured quadrupole, in particular: $(\delta T)_{I}^{2} \simeq 1252 \mu \mathrm{K}^{2}$. In such a case the anisotropic contribution could help in reducing the value of the quadrupole for certain values of the phases and fluid velocities. Once again the first condition is automatically satisfied, and the second condition yields

$$
\begin{aligned}
& 54 \mu \mathrm{K}^{2} \lesssim\left(\delta T_{A}\right)^{2} \lesssim 3857 \mu \mathrm{K}^{2} \quad 68 \% \text { C.L., } \\
& 0 \mu \mathrm{K}^{2} \lesssim\left(\delta T_{A}\right)^{2} \lesssim 9256 \mu \mathrm{K}^{2} \quad 95 \% \text { C.L. }
\end{aligned}
$$

Notice that the $95 \%$ confidence interval includes the standard prediction from inflation and for that reason the lower limit vanishes in that case in (47). According to these results, for certain orientations of the velocities and the values of the phase factors, $Q_{A}$ could lower the value of quadrupole and make it compatible with the observed one even at the $1 \sigma$ level. We will compare these limits with the predictions from several models, but before that we need to extend our calculations beyond the perturbative regime.

\section{FAST-MOVING FLUIDS: EXACT EQUATIONS}

In the previous section we have studied the problem of obtaining the quadrupole produced by the fact that dark energy does not share a common rest frame with matter and radiation. To that end, we have used cosmological perturbation theory to compute the metric perturbations by means of the simple formula (29), valid when the velocities are small. Such formula could also be reasonably useful for high initial velocities provided they drop in time and rapidly reach the perturbative regime. If we look at Eq. (30) we conclude that this condition is satisfied if $w_{\alpha}<$ $\frac{1}{3}$. Besides, if $w_{\alpha}=\frac{1}{3}$, as in the radiation case, the velocity is constant so we just need to have a small initial velocity. However, some models have been proposed in which the total energy density of the Universe could contain in certain epochs a non-negligible contribution of fluids with equation of state such that $w_{\alpha}>\frac{1}{3}$. This is for instance the case of stiff-fluid cosmologies or some tracking dark energy models. In those cases the velocities grow in time, perturbation theory will eventually break down at some point and it becomes necessary to solve the exact problem.

In order to simplify the equations in this case, we shall change the gauge used in the previous section by one in which $g_{00}=1$, keeping the condition $g_{0 i}=0$. Moreover, we shall also assume that the fluids are moving along the 
$z$-axis with no rotation which means that axisymmetry holds. The most general metric having that symmetry with this gauge choice can be written as follows:

$$
d s^{2}=d t^{2}-a_{\perp}^{2}\left(d x^{2}+d y^{2}\right)-a_{\|}^{2} d z^{2} .
$$

On the other hand, the energy-momentum tensor for each fluid reads:

$$
\begin{aligned}
& T_{\alpha 0}^{0}=\gamma_{\alpha}^{2}\left(\rho_{\alpha}+p_{\alpha}\right)-p_{\alpha}, \\
& T_{\alpha 0}^{i}=\gamma_{\alpha}^{2}\left(\rho_{\alpha}+p_{\alpha}\right) v_{z \alpha} \delta^{i z}, \\
& T_{\alpha i}^{0}=-\gamma_{\alpha}^{2}\left(\rho_{\alpha}+p_{\alpha}\right) a_{\|}^{2} v_{z \alpha} \delta_{i z}, \\
& T_{\alpha j}^{i}=-\gamma_{\alpha}^{2}\left(\rho_{\alpha}+p_{\alpha}\right) a_{\|}^{2} v_{z \alpha}^{2} \delta^{i z} \delta_{j z}-p_{\alpha} \delta_{j}^{i} .
\end{aligned}
$$

Note that the velocities appearing in these expressions are no longer the same as those of the previous section since, here, we have defined them as derivatives with respect to the time $t$ not with respect to $\eta$. However, it is easy to translate these velocities into the others just by defining a mean scale factor $a \equiv \sqrt[3]{a_{\perp}^{2} a_{\|}}$because $d t \simeq a d \eta$ and, therefore, $\frac{d x^{i}}{d \eta} \simeq a \frac{d x^{i}}{d t}$, which is a good approximation in the perturbative regime. Moreover, we have to notice that for an appropriate definition of fluid velocity, we have to rescale $V \equiv a_{\|} v$, so that $V^{2} \leq 1$.

Now, it is convenient to introduce the variables $\theta_{\alpha}$ defined by $\cosh \theta_{\alpha}=\gamma_{\alpha}$ so that the equations adopt a simpler structure. Velocities are related to $\theta_{\alpha}$ by means of $\tanh \theta_{\alpha}=a_{\|} v_{\alpha}$. With these new variables, the Einstein equations from (48) and (49) take the form

$$
\begin{aligned}
& H_{\perp}^{2}+2 H_{\perp} H_{\|}=8 \pi G \sum_{\alpha}\left(\cosh ^{2} \theta_{\alpha}+w_{\alpha} \sinh ^{2} \theta_{\alpha}\right) \rho_{\alpha}, \\
& \dot{H}_{\perp}+\dot{H}_{\|}+H_{\perp}^{2}+H_{\|}^{2}+H_{\perp} H_{\|}=-8 \pi G \sum_{\alpha} p_{\alpha}, \\
& 2 \dot{H}_{\perp}+3 H_{\perp}^{2}=-8 \pi G \sum_{\alpha}\left(w_{\alpha} \cosh ^{2} \theta_{\alpha}+\sinh ^{2} \theta_{\alpha}\right) \rho_{\alpha},
\end{aligned}
$$

where ${ }^{\bullet} \equiv \frac{d}{d t}$ and $H_{\perp} \equiv \dot{a}_{\perp} / a_{\perp}, H_{\|} \equiv \dot{a}_{\|} / a_{\|}$are the transverse and longitudinal expansion rates, respectively. These equations reduce to the Friedmann ones when $a_{\perp}=a_{\|}$and $v_{z \alpha}=0$. Again, as in the perturbative case, we need some extra equations to close the problem which are those given by the independent energy-momentum tensor conservation. These equations can be written as follows:

$$
\begin{gathered}
\dot{v}_{z \alpha}=-\frac{\left(\left(w_{\alpha}-1\right) \cosh ^{2} \theta_{\alpha}-1\right) H_{\|}+2 w_{\alpha} H_{\perp}}{\left(w_{\alpha}-1\right) \cosh ^{2} \theta_{\alpha}-w_{\alpha}} v_{z \alpha} \\
\dot{\rho}_{\alpha}=\frac{\left(1+w_{\alpha}\right)\left(H_{\|}+2 H_{\perp} \cosh ^{2} \theta_{\alpha}\right)}{\left(w_{\alpha}-1\right) \cosh ^{2} \theta_{\alpha}-w_{\alpha}} \rho_{\alpha}
\end{gathered}
$$

Besides, one can find the following equations for the evolution of $\theta_{\alpha}$ :

$$
\dot{\theta}_{\alpha}=-\frac{\left(w_{\alpha}-1\right) H_{\|}+2 w_{\alpha} H_{\perp}}{\left(w_{\alpha}-1\right) \cosh ^{2} \theta_{\alpha}-w_{\alpha}} \sinh \theta_{\alpha} \cosh \theta_{\alpha} .
$$

The spatial geodesic equations for the metric considered are

$$
\begin{aligned}
& \frac{d^{2} x}{d \lambda^{2}}+2 H_{\perp} \frac{d t}{d \lambda} \frac{d x}{d \lambda}=0, \\
& \frac{d^{2} y}{d \lambda^{2}}+2 H_{\perp} \frac{d t}{d \lambda} \frac{d y}{d \lambda}=0 \\
& \frac{d^{2} z}{d \lambda^{2}}+2 H_{\|} \frac{d t}{d \lambda} \frac{d z}{d \lambda}=0
\end{aligned}
$$

where $\lambda$ is an affine parameter. The first integral for these equations is given by

$$
\frac{d \vec{r}}{d \lambda}=\left(\frac{n_{x}}{a_{\perp}^{2}}, \frac{n_{y}}{a_{\perp}^{2}}, \frac{n_{z}}{a_{\|}^{2}}\right)
$$

being $\vec{r}=(x, y, z)$ and the integration constants can be chosen for simplicity in such a way that $\vec{n}^{2}=1$. Moreover, from the condition of null geodesic we get

$$
\frac{d t}{d \lambda}=\sqrt{\frac{n_{\perp}^{2}}{a_{\perp}^{2}}+\frac{n_{\|}^{2}}{a_{\|}^{2}}}
$$

with $n_{\perp}^{2}=n_{x}^{2}+n_{y}^{2}$ and $n_{\|}^{2}=n_{z}^{2}$. Then, for an observer with velocity $u^{\mu}=\gamma(1, \vec{v})$ the energy of the photon is

$$
\mathcal{E}=\gamma E\left[\sqrt{\frac{n_{\perp}^{2}}{a_{\perp}^{2}}+\frac{n_{\|}^{2}}{a_{\|}^{2}}}-\vec{n} \cdot \vec{v}\right]
$$

and the corresponding temperature fluctuation reads

$$
\frac{\delta T}{T}=\frac{\gamma_{0} a_{0}}{\gamma_{\mathrm{dec}} a_{\mathrm{dec}}} \frac{\sqrt{\frac{n_{\perp}^{2}}{a_{\perp 0}^{2}}+\frac{n_{\|}^{2}}{a_{\| 0}^{2}}}-\vec{n} \cdot \vec{v}_{0}}{\sqrt{\frac{n_{\perp}^{2}}{a_{\perp \mathrm{dec}}^{2}}+\frac{n_{\|}^{2}}{a_{\| \mathrm{dec}}^{2}}}-\vec{n} \cdot \vec{v}_{\mathrm{dec}}}-1
$$

where again the indices 0 and dec denote the present and decoupling times, respectively.

\section{MODEL EXAMPLES}

\section{A. Constant equation of state}

The simplest dark energy model we will consider is that corresponding to a fluid with constant equation of state $w_{\mathrm{DE}} \simeq-1$. Note that in the case $w_{\mathrm{DE}}=-1$, i.e. pure cosmological constant, dark energy does not contribute to the center of mass velocity (6) and, therefore, the center of mass frame agrees with the radiation frame. This means that all the fluids would share a common rest frame and no effects on the CMB would be possible. When $w_{\mathrm{DE}}$ is close to -1 , the velocity of dark energy scales as $\sim a^{-4}$ and its energy density is nearly constant (see Fig. 1). Since dark 

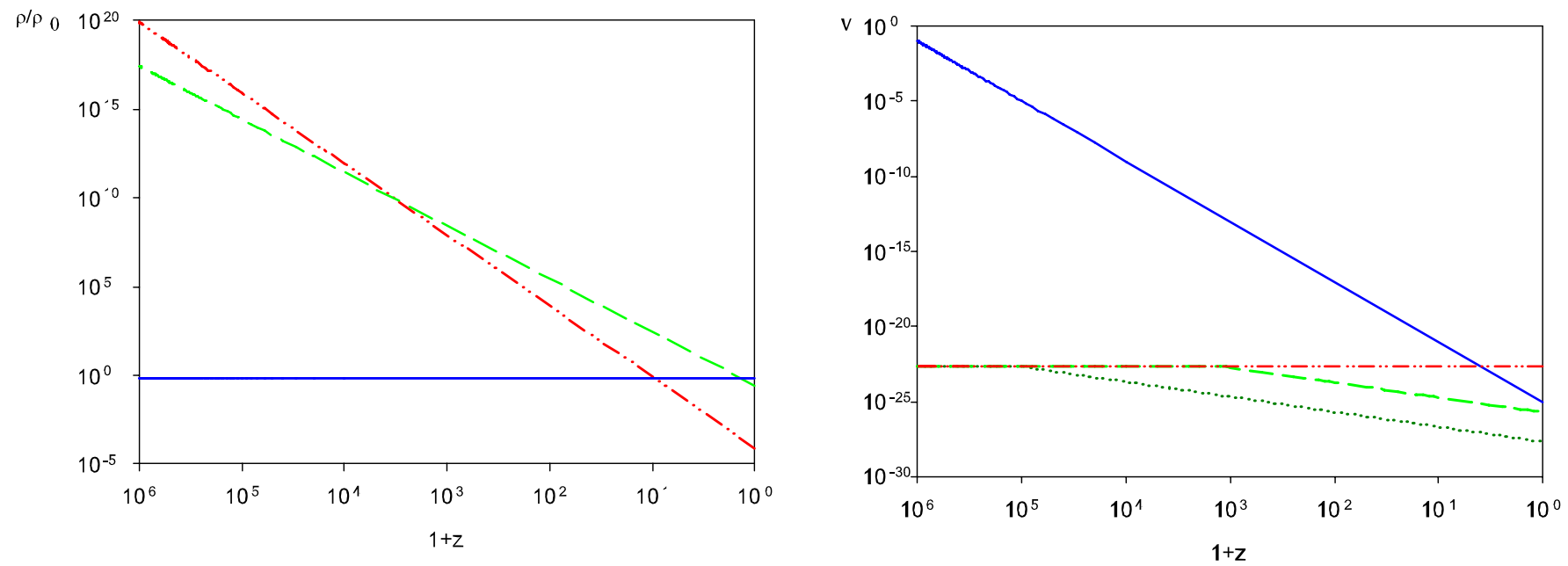

FIG. 1 (color online). Evolution of densities and velocities in a model with constant equation of state as described in the text. Continuous line (blue) for dark energy, dashed-dotted line (red) for radiation, dotted line (cyan) for dark matter, and dashed line (green) for baryonic matter. On the left baryonic and dark matter are added together and plotted in a dashed line (green). Notice that in this plot dark matter is assumed to decouple at $z \simeq 10^{5}$.

energy velocity decreases very fast, its contribution to the quadrupole is very small. Besides, the velocity of radiation (and therefore that of matter) is determined by the initial dark energy velocity and the gauge condition (31) as

$$
\vec{v}_{R}=\frac{1+w_{\mathrm{DE}}}{1+w_{R}} \frac{\Omega_{\mathrm{DE}}}{\Omega_{R}} a_{*}^{4} \vec{v}_{\mathrm{DE}}^{*},
$$

where $\vec{v}_{\mathrm{DE}}^{*}$ is the initial dark energy velocity. Taking $\Omega_{\mathrm{DE}}=0.73, \Omega_{R}=8.18 \times 10^{-5}, w_{\mathrm{DE}}=-0.97$, and $a_{*} \sim$ $10^{-6}$, we get $\vec{v}_{R} \simeq 2 \times 10^{-22} \vec{v}_{\mathrm{DE}}^{*}$. The value of $a_{*}$ taken corresponds to a favorable case since lower values would lead to much lower velocities of radiation and matter. Then, even for initial velocities of dark energy close to 1 , the velocities of matter and radiation are extremely small, which means that the three fluids are very nearly at rest in the cosmic center of mass frame. That way, the quadrupole generated in this model is totally negligible.

\section{B. Scaling models}

Scaling models [22] are those with equation of state such that dark energy mimics the dominant component of the Universe throughout most of the Universe evolution. Thus, dark energy evolves as radiation before matter-radiation equality and as matter after that. However, in order to explain the accelerated expansion of the Universe, dark energy has to exit from that regime and join into one with $w_{\text {DE }}<-1 / 3$ at some point. Then, the evolution of the dark energy density is given by

$$
\rho_{\mathrm{DE}}= \begin{cases}\rho_{\mathrm{DE} 0} a_{T}^{-3 w_{\mathrm{DE}}} a_{e q} a^{-4} & a<a_{e q} \\ \rho_{\mathrm{DE} 0} a_{T}^{-3 w_{\mathrm{DE}}} a^{-3} & a_{e q}<a<a_{T}, \\ \rho_{\mathrm{DE} 0} a^{-3\left(w_{\mathrm{DE}}+1\right)} & a>a_{T}\end{cases}
$$

where as commented before, $a_{T}$ is the scale factor when dark energy leaves the scaling regime and $\rho_{\mathrm{DE} 0}$ is the present value of the dark energy density.

In the evolution of dark energy velocity, we have to take into account the momentum conservation equation given (to first order) by (15). This equation implies that the dark energy velocity must be discontinuous at the transition points since the equation of state jumps at those times whereas the quantity $a^{4}\left(1+w_{\mathrm{DE}}\right) \rho_{\mathrm{DE}} \vec{v}_{\mathrm{DE}}$ is constant, being $\rho_{\mathrm{DE}}$ continuous. With this in mind, we get the following evolution for dark energy velocity:

$$
\vec{v}_{\mathrm{DE}}= \begin{cases}\vec{v}_{\mathrm{DE}}^{*} & a<a_{e q} \\ \frac{4}{3} a_{e q} a^{-1} \vec{v}_{\mathrm{DE}}^{*} & a_{e q}<a<a_{T} . \\ \frac{4 a_{e q} a_{T}^{-3 w_{\mathrm{DE}}}}{3\left(1+w_{\mathrm{DE}}\right)} a^{3 w_{\mathrm{DE}}-1} \vec{v}_{\mathrm{DE}}^{*} & a>a_{T}\end{cases}
$$

The discontinuities in the velocity arise because we are considering abrupt changes in the equation of state. If these changes were smooth, the results would be essentially unaffected since the final values of the velocities would remain those in (63). In Fig. 2, we show the evolution of the energy densities and velocities for a typical scaling model.

We can see from the previous expression that, in the second transition, the closer $w_{\mathrm{DE}}$ is to -1 , the more the velocity grows after the transition. The case $w_{\mathrm{DE}}=-1$ is not divergent because, if that was the case, the conservation equation would become trivial and the velocity evolution got from (30) would not make sense anymore.

In these scaling models, the first transition can be set at the matter-radiation equality and the second one must be chosen such that we get the observed dark energy density today. Moreover, the initial velocity of radiation, and therefore that of matter, is fixed by the initial velocity of dark energy via the gauge condition (31). Because matter is subdominant with respect to radiation before equality, the 

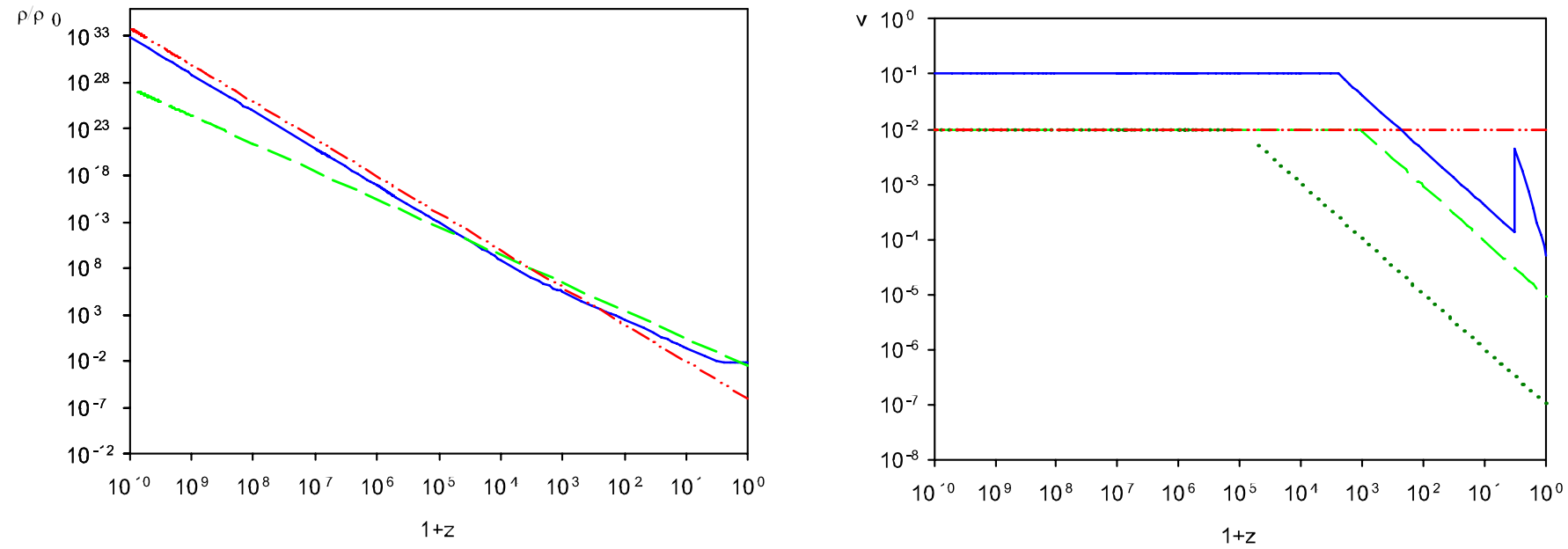

FIG. 2 (color online). Densities and velocities evolution in a scaling model with $v_{\mathrm{DE}}^{*}=0.1$ and $\epsilon=0.1$. As in the previous figure, the continuous line (blue) is for dark energy, dashed-dotted line (red) for radiation, dotted line (cyan) for dark matter, and dashed line (green) for baryonic matter. On the left baryonic and dark matter are added together and plotted in a dashed line (green). Notice that in this plot dark matter is also assumed to decouple at $z \simeq 10^{5}$ and $a_{*}=10^{-10}$.

matter contribution in (31) can be neglected and we obtain that

$$
\vec{v}_{R}^{*}=-\epsilon \vec{v}_{\mathrm{DE}}^{*}
$$

where $\epsilon \equiv \rho_{\mathrm{DE}}\left(a_{*}\right) / \rho_{R}\left(a_{*}\right)$ is the initial dark energy density fraction (neglecting the matter contribution). Notice that this fraction does not depend on $a_{*}$, because dark energy scales as radiation in the radiation-dominated era. Then, we can obtain a relation between $a_{T}$ and $\epsilon$ just by computing that quotient from the known expressions for the energy densities evolutions of each fluid. When doing that it results:

$$
a_{T}=\left[\frac{\Omega_{\mathrm{DE}} a_{e q}}{\Omega_{R} \epsilon}\right]^{1 / 3 w_{\mathrm{DE}}} .
$$

Since we need $w_{\mathrm{DE}}\left(z_{T}\right)<-1 / 3$ in order to have accelerated expansion, we see from the previous formula that $a_{T}$ grows as $\epsilon$ grows, more precisely if we take $w_{\mathrm{DE}}(z<$ $\left.z_{T}\right)=-0.97$, then $a_{T} \propto \epsilon^{0.34}$. Since primordial nucleosynthesis imposes an upper limit on $\epsilon$, we can establish also an upper limit on $a_{T}$ just by setting the maximum value of $\epsilon$ on (65). This maximum value is $\epsilon_{\max } \simeq 0.2$, (see for instance [23]) so we get the constraint $a_{T} \lesssim 0.41$ for this kind of scaling models where we have taken $\Omega_{\mathrm{DE}}=0.73$, $\Omega_{R}=8.18 \times 10^{-5}$, and $a_{e q}=\frac{1}{3300}$.

By inserting the corresponding values for the densities and velocities of the four fluids, as well as the equation of state considered into (33), we can compute the quadrupole produced by the relative motion of the fluids. For our calculations we shall take $\Omega_{B}=0.046, \Omega_{\mathrm{DM}}=0.23$, $a_{\mathrm{dec}}=\frac{1}{1100}$, and $w_{\mathrm{DE}}\left(z<z_{T}\right)=-0.97$ and the values given above. Figure 3 shows the evolution of the contribution of each fluid to the metric perturbation $h_{\alpha}$. We can see that the typical behavior is a rapid growth during the radiation era to reach finally a slightly growing regime in the matter era (notice that the dependence on $a_{*}$ is only logarithmic). In spite of the fact that the perturbation is $\mathcal{O}\left(v_{\mathrm{DE}}^{* 2}\right)$, the quadrupole is expected to be smaller because $h$ barely grows in the epoch since decoupling to today and, as we mentioned in Sec. I, the quadrupole is essentially given by the growth of the perturbation during that epoch.

The quadrupole produced by scaling models is fixed by two parameters: the initial velocity $v_{\mathrm{DE}}^{*}$ and the initial energy ratio $\epsilon$ of dark energy. It is easy to see from (33) that $h_{\alpha}$ and, therefore the quadrupole, is proportional to $v_{\mathrm{DE}}^{* 2}$. Obviously, this dependence is valid just for small

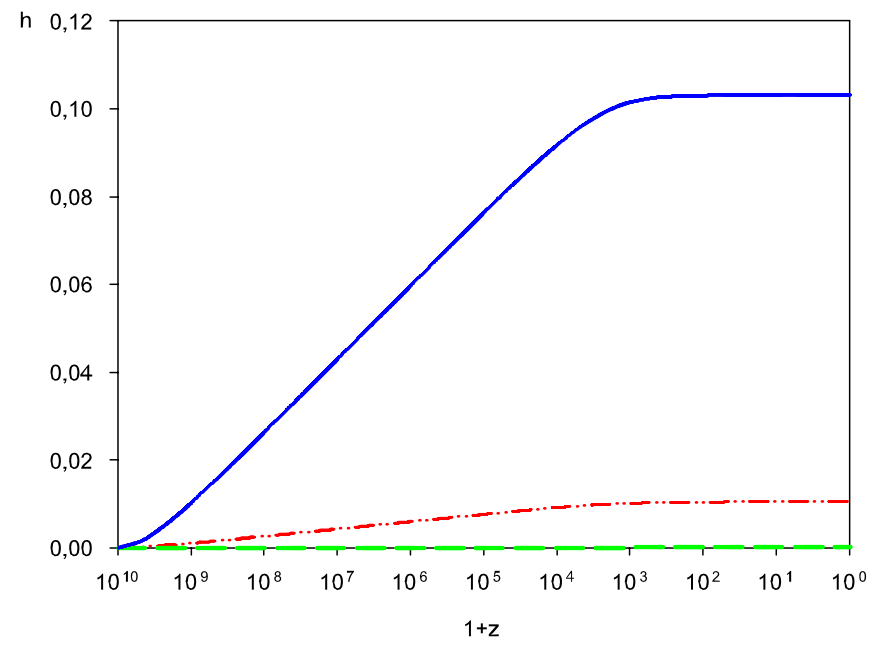

FIG. 3 (color online). Evolution of metric perturbations due to each fluid in a scaling model with $\epsilon=0.1$ and $v_{*}=0.1$. We can see that the largest contribution comes from dark energy (continuous blue line) and matter (dashed green line) essentially does not contribute. Radiation is shown with a dashed-dotted (red) line. 
velocities since when we consider velocities close to the speed of light we have to take into account relativistic effects. The dependence of the quadrupole on $\epsilon$ can be found to be linear for $\epsilon \lessgtr 0.07$ with a slope 0.44 so we can conclude that the quadrupole is very well approximated by the simple expression

$$
Q_{A} \simeq 0.44 \epsilon v_{\mathrm{DE}}^{* 2} .
$$

As commented before, this expression is valid only for small velocities. According to the bounds on the quadrupole obtained in (46) and (47), there are allowed regions in the parameter space $\left(\epsilon, v_{\mathrm{DE}}^{*}\right)$, which from (66) are limited by the curves $\epsilon=k_{ \pm} / v_{*}^{2}$ where the constants $k_{ \pm}$correspond to the upper and lower limits on $Q_{A}$. In Fig. 4 we show these regions obtained numerically with the exact equations (see Sec. IV). As we said above, the second order calculation is a good approximation for velocities lower than 0.1. However, when the velocities are large (close to 1) values of $\epsilon \lessgtr \mathcal{O}\left(10^{-6}\right)$ are necessary in order to explain the observed quadrupole. Notice once again that these regions have been obtained in the case in which the measured quadrupole has two contributions, one coming from inflation and a second contribution coming from the fluids motion.

To end this section we shall show why we can neglect the third term in (27) with respect to that containing the metric perturbation $h_{i j}$. Let us recall that term:

$$
\left.(\vec{v} \cdot \vec{n})\right|_{\mathrm{dec}} ^{0}\left(\vec{v}_{\mathrm{dec}} \cdot \vec{n}\right)
$$

The first factor in this expression is nothing but the dipole which is $\sim 10^{-3}$. The second factor contains the velocity of the observer at decoupling time which coincides with matter velocity (and therefore with that of radiation) at

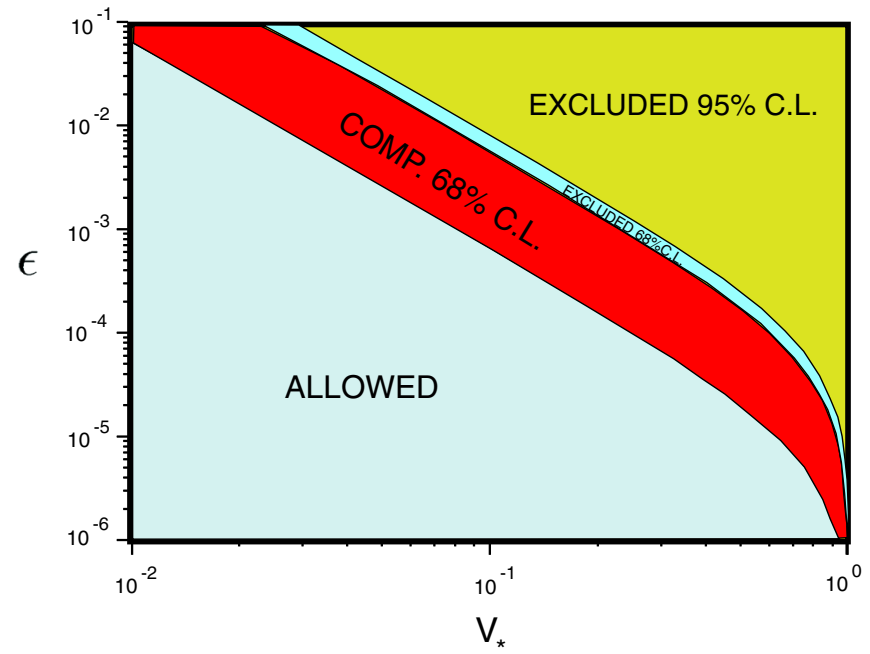

FIG. 4 (color online). Exclusion plot in the parameter space $\left(\epsilon, v_{\mathrm{DE}}^{*}\right)$ for a scaling dark energy model. The allowed region corresponds to the limits given in (46). The dark (red) strip corresponds to the regions for which $Q_{A}$ could explain the observed quadrupole at the $68 \%$ C.L. according to (47). that moment. Then, if we recall the relation (64) between radiation and dark energy velocities, we find that this term is $\sim 10^{-3} \epsilon v_{\mathrm{DE}}^{*}$. On the other hand, the last term in (27) is $\sim \epsilon v_{\mathrm{DE}}^{* 2}$ as we have just seen above. Hence, if we call $Q_{v}$ and $Q_{h}$ to the last two terms in (27), respectively, we have that $Q_{h} \sim 10^{3} v_{\mathrm{DE}}^{*} Q_{v}$ and we see that $Q_{v}$ will be larger than $Q_{h}$ only for $v_{\mathrm{DE}}^{*}<10^{-3}$. However, in such a case the contribution to the quadrupole is $\lesssim 10^{-6} \epsilon$ which is negligible.

\section{Tracking models}

In this section we would like to comment on the difficulties which can appear in certain dark energy models when we consider perturbations in the fluids velocities. In general, any model with a stiff stage in which its equation of state satisfies $w>\frac{1}{3}$, would be unstable with respect to velocity perturbations according to (30). This could be the case of certain tracking models [24]. These are models in which the energy density of dark energy follows a common evolutionary track for a wide range of initial conditions. This attractor behavior makes these kinds of models an interesting alternative to a cosmological constant since they alleviate the so-called coincidence problem. Unlike scaling models, in this case dark energy does not necessarily mimic the dominant component. In the model proposed in [24] the equation of state is initially close to 1 , then it changes to -1 and, finally, it oscillates around -0.2 . Figure 5 shows a typical behavior when $w>\frac{1}{3}$ : first the velocity perturbation (defined as $V \equiv a_{\|} v$ ) grows according to (30) and asymptotically approaches 1 . We can understand this from the exact conservation equations (53) by taking the ultrarelativistic limit $\theta \gg 1$. This yields the solutions

$$
v=v_{0} a_{\|}^{-1}, \quad \rho=\rho_{0} a_{\perp}^{-2((1+w) /(1-w))} .
$$

This means that $V \simeq 1$ is a solution of the equations. In addition, the energy density for $w>1 / 3$ falls very fast with the expansion when compared with the usual behavior $\rho=\rho_{0} a^{-3(1+w)}$. In addition, $\gamma^{2} \rho$, which is the quantity that contributes to the Hubble rate in (50), decays as $\left(a_{\|} a_{\perp}\right)^{-2}$, once the fluid reaches the ultrarelativistic regime, regardless of the value of $w$.

In the limiting case of stiff fluids with $w=1$, it is possible to obtain exact solutions. Thus the velocity perturbation and the energy density are

$$
V=V_{0} a_{\perp}^{2}, \quad \rho=\rho_{0} \frac{e^{-4 \int H_{\perp} \cosh ^{2} \theta d t}}{a_{\|}^{2}} .
$$

Thus, the velocity of the fluid grows as $a_{\perp}^{2}$ until it reaches the speed of light in a finite time and the density falls to zero at the same time because $\theta$ becomes infinity at that moment. From that time on, the fluid will keep moving at the speed of light with vanishing energy density. Notice 

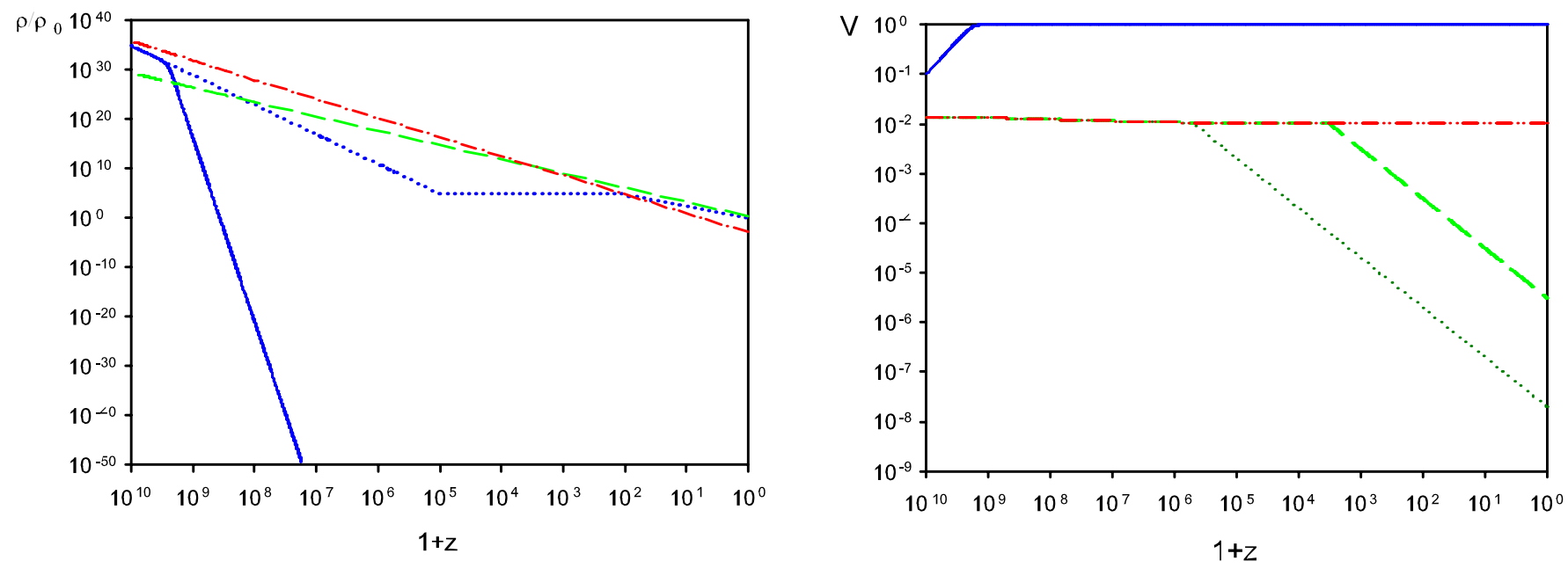

FIG. 5 (color online). Densities and velocities evolution in a typical tracking model with an initial equation of state $w_{\mathrm{DE}}=0.9$, which changes to $w_{\mathrm{DE}}=-1$ and then to $w_{\mathrm{DE}} \sim-0.2$. The continuous line (blue) is for dark energy in the model with moving dark energy, whereas the blue dotted line is for static dark energy, dashed-dotted line (red) for radiation, dashed line (green) for baryonic and dark matter. We see that when $V_{\mathrm{DE}}$ reaches 1 , the corresponding density begins falling too fast to be able to recover the present value for $\Omega_{\mathrm{DE}}$.

that $\gamma$ becomes infinity in such a way that the momentum is conserved i.e. the matching between the two regimes must be taken so that $\gamma^{2} \rho$ is finite and continuous. In any case, we see that when the velocity is high enough the density falls to zero and, the closer $w$ is to one, the faster the fall of the density is, so that we cannot recover the present value for the dark energy density. Notice that this general behavior is independent of the value of the initial velocity and, accordingly, even in models in which all the fluids are initially at rest, a small perturbation in the velocity could change dramatically the final values of the densities, unless fine-tunings of the transition redshifts are introduced.

\section{Null dark energy}

In this section we shall study the case in which dark energy behaves as a null fluid, whose energy-momentum tensor reads

$$
T_{N}^{\mu \nu}=\left(\rho_{N}+p_{N}\right) l^{\mu} l^{\nu}-p_{N} g^{\mu \nu}
$$

with $l^{\mu}$ a null vector given, in the Bianchi type I metric (48), by $l^{\mu}=\left(1,0,0, a_{\|}^{-1}\right)$. For this kind of fluid, the conservation of energy and momentum can be expressed as follows:

$$
\begin{gathered}
0=\dot{p}_{N}, \\
0=\left(\dot{\rho}_{N}+\dot{p}_{N}\right)+2\left(H_{\|}+H_{\perp}\right)\left(\rho_{N}+p_{N}\right) .
\end{gathered}
$$

These equations imply that the pressure is constant and that $\left(\rho_{N}+p_{N}\right)$ scales as $\left(a_{\|} a_{\perp}\right)^{-2}$, so that the energy density is given by $\rho_{N}=\rho_{N 0}\left(a_{\|} a_{\perp}\right)^{-2}-p_{N 0}$ where $p_{N 0}$ and $\rho_{N 0}$ are constants of integration. Since the anisotropy is expected to be small, the energy density of this fluid behaves as radiation during the early epoch and as a cosmological constant with energy density $-p_{N 0}$ at late times. Now, if we require $\rho_{N}$ to be positive at all times, we conclude that the pressure must be negative, as corresponds to a cosmological constant. Notice that this is a general result for any null fluid whose energy-momentum tensor is given by (70). The transition between both regimes can be easily calculated and it is given by $a_{T} \simeq\left(-\frac{\rho_{N 0}}{p_{N 0}}\right)^{1 / 4}$. Since $p_{N 0}=$ $-0.73 \rho_{c 0}$, where $\rho_{c 0}$ is the critical density today, we have that $a_{T}=0.1 \epsilon^{1 / 4}$ where $\epsilon \equiv \frac{\rho_{N 0}}{\rho_{R 0}}$ is the ratio of dark energy density with respect to radiation which is almost constant. Besides, this ratio is also the initial contribution of dark energy to the total energy density which has an upper limit imposed by primordial nucleosynthesis. Taking once again $\epsilon_{\max } \lesssim 0.2$, we have an upper limit on the transition given by $a_{T} \lesssim 2 \times 10^{-2}$.

The exact Einstein equations in this case are

$$
\begin{aligned}
H_{\perp}^{2}+2 H_{\perp} H_{\|}= & 8 \pi G \sum_{\alpha}\left(\cosh ^{2} \theta_{\alpha}+w_{\alpha} \sinh ^{2} \theta_{\alpha}\right) \rho_{\alpha} \\
& +8 \pi G \rho_{N}, \\
\dot{H}_{\perp} & +\dot{H}_{\|}+H_{\perp}^{2}+H_{\|}^{2}+H_{\perp} H_{\|} \\
= & -8 \pi G \sum_{\alpha} p_{\alpha}-8 \pi G p_{N}, \\
2 \dot{H}_{\perp}+3 H_{\perp}^{2}= & -8 \pi G \sum_{\alpha}\left(w_{\alpha} \cosh ^{2} \theta_{\alpha}+\sinh ^{2} \theta_{\alpha}\right) \rho_{\alpha} \\
& -8 \pi G\left(\rho_{N}+2 p_{N}\right),
\end{aligned}
$$

where now $\alpha=B, D M, R$. Moreover, we still have the gauge condition $\vec{S}=0$ which yields the following constraint: 


$$
\sum_{\alpha} \gamma_{\alpha}^{2}\left(\rho_{\alpha}+p_{\alpha}\right) v_{\alpha}+\left(\rho_{N}+p_{N}\right)=0 .
$$

In the radiation-dominated era we can neglect the contribution of matter (dark matter and baryons) to the latter sum, so we get

$$
\gamma_{R}^{2} v_{R}=-\frac{\rho_{N}+p_{N}}{\rho_{R}+p_{R}} .
$$

Since, in that epoch, dark energy must be subdominant with respect to radiation, the quotient on the RHS is small and, therefore, the velocity of radiation is also small. This allows us to consider the perturbative regimen in the velocities (except, obviously for the null fluid).

Therefore, if we assume that the anisotropy generated is small we can set the following form for $a_{\|}$and $a_{\perp}$ :

$$
a_{\perp}=a\left(1+\delta_{\perp}\right), \quad a_{\|}=a\left(1+\delta_{\|}\right) .
$$

With this ansatz it is easy to see that $h=2\left(\delta_{\|}-\delta_{\perp}\right)$. Then, inserting (78) in (75) and expanding up to first order in $\delta$ 's and $v_{\alpha}$ we can get the following equation for $h$ :

$$
\frac{d}{d t}\left(a^{3} \frac{d h}{d t}\right)=2 a^{3}\left(\rho_{N}+p_{N}\right) .
$$

This equation can be easily solved by means of two direct integrations and its solution can be expressed as follows:

$$
h=6 \int_{a_{*}}^{a} \frac{1}{\tilde{a}^{4}}\left[\int_{a_{*}}^{\hat{a}} \hat{a}^{2}\left(\rho_{N}+p_{N}\right) \frac{d \hat{a}}{\sqrt{\sum_{\alpha} \rho_{\alpha}}}\right] \frac{d \tilde{a}}{\sqrt{\sum_{\alpha} \rho_{\alpha}}} .
$$

In principle, the problem is not solved yet since $\rho_{N}+p_{N}$ depends on $\delta_{\|}$and $\delta_{\perp}$. However, we can consider the lowest order in this quantity, i.e., $\rho_{N}+p_{N}=\rho_{N 0} a^{-4}$ to obtain the dominant contribution to the quadrupole. This is

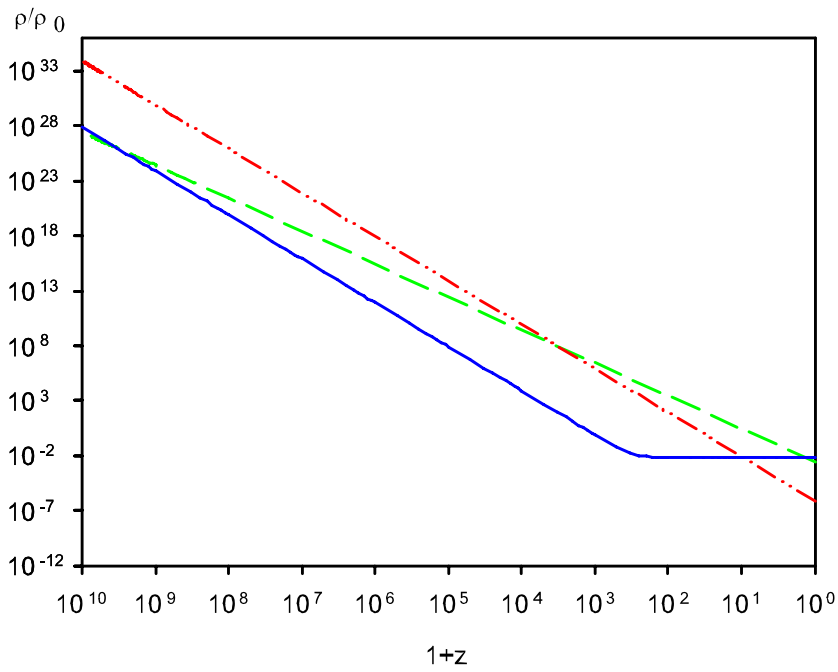

justified because $\frac{\rho_{N 0}}{\rho_{R 0}}$ is of the same order as $v_{R}$ as we can see from (77). We have to note that, to this order, the quadrupole depends just on the null fluid because the first contribution to the anisotropy due to the rest of the fluids is of second order in the velocities, whereas the null fluid contributes to first order. In Fig. 6 we plot the evolution of the fluids densities and $h$ function for a null fluid with $\epsilon=$ $5 \times 10^{-6}$.

In this model we only have one free parameter: $\rho_{N 0}$ or, equivalently, $\epsilon \equiv \frac{\rho_{N 0}}{\rho_{R 0}}$, so we can get bounds on $\epsilon$ just from (46) and (47). Besides, the quadrupole is linear in $\epsilon$, as we see looking at (80), more precisely we have that the quadrupole is given by $Q_{A} \simeq 2.58 \epsilon$. Now again the contribution from the term $Q_{v}$ given by (67) is negligible compared with $Q_{A}$ since $Q_{v} \simeq 10^{-3} \epsilon$.

This expression is nearly independent of $a_{*}$ because the quadrupole depends on the difference $h_{0}-h_{\mathrm{dec}}$ which is not very sensitive to the time at which we set the initial conditions. Comparing the expression obtained for the quadrupole with the previous bounds, we get that the allowed region in (46) corresponds to

$$
\begin{array}{ll}
\epsilon & \leqslant 6.1 \times 10^{-6} \quad 68 \% \text { C.L. } \\
\epsilon \lesssim 1.1 \times 10^{-5} & 95 \% \text { C.L. }
\end{array}
$$

whereas for

$$
\begin{gathered}
1 \times 10^{-6} \lesssim \epsilon \lessgtr 8.8 \times 10^{-6} \quad 68 \% \text { C.L. } \\
0 \leqq \epsilon \lesssim 1.4 \times 10^{-5} \quad 95 \% \text { C.L. }
\end{gathered}
$$

the null fluid could make the predicted quadrupole to agree with observations, as shown in (47).

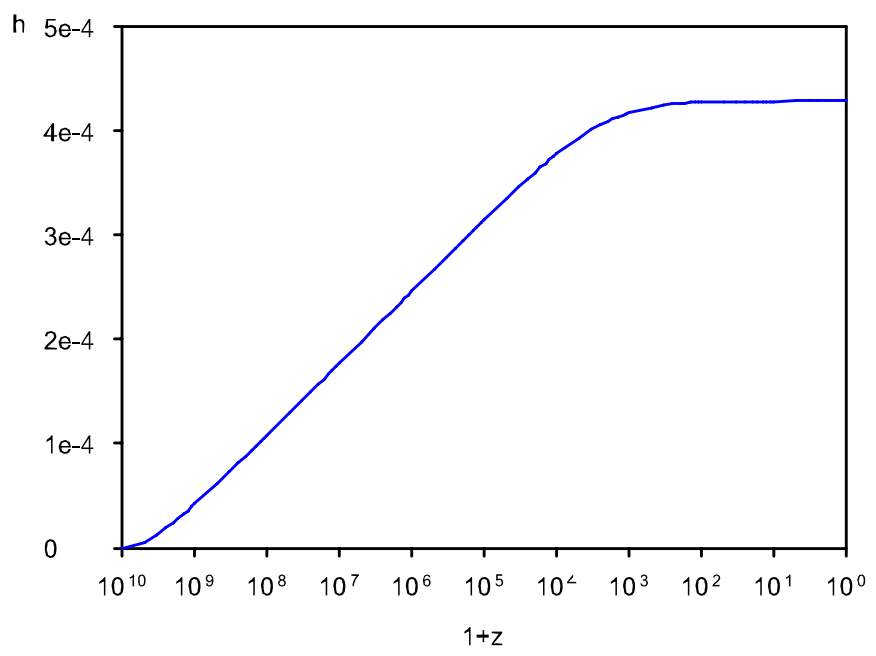

FIG. 6 (color online). Left: densities evolution for a null fluid with $\epsilon=5 \times 10^{-6}$. Matter is plotted with dashed (green) line, dotteddashed (red) line for radiation, and continuous (blue) line for dark energy. We see that the null fluid behaves as in a scaling model except during the matter dominated epoch. Right: evolution of $h$ showing that the anisotropy grows up to a maximum value where it keeps almost constant. 


\section{CONCLUSIONS AND DISCUSSION}

In this paper we have studied homogeneous models of dark energy in which the rest frame of the different fluids can differ from each other. We have considered the evolution of slow-moving and fast-moving fluids and shown that, starting from an initially isotropic universe, the fluids motions can generate an anisotropic expansion in which the anisotropy degree typically grows in time. Such anisotropies are shown to contribute to the CMB dipole and quadrupole only, but not to higher multipoles. We apply those results to some dark energy models and find that in models with constant equation of state, even for initial velocities of dark energy close to the speed of light, throughout the matter era all the fluids would practically share a common rest frame and therefore no effect on the quadrupole is expected. However, in the case of scaling models it is shown that the anisotropy grows during the radiation era and reaches an almost constant value during matter domination. The effect on the CMB quadrupole can be relevant and bounds on the velocity and initial fraction of dark energy can be found. We also find that for models with an initial stage in which the equation of state is stiffer than radiation, as for instance in some tracking models, the velocity approaches the speed of light whereas the energy density decays faster than in the case in which dark energy is at rest with respect to matter and radiation. This fact spoils the predictions of those models for the density parameters at late times. Finally we have considered also fluids moving at the speed of light and found that generi- cally they behave as a cosmological constant at late time, provided their energy density is positive at all times, whereas they act as radiation at early times. The contribution to the quadrupole is also used to set limits on the relative contribution of dark energy in the radiationdominated era.

The models presented in this paper with moving fluids lead to Bianchi I anisotropic metrics of the type recently studied in [21]. However unlike that model with decaying anisotropies generated by the presence of magnetic fields in the early universe, in our work the motion of dark energy supports the anisotropies which could have a nonnegligible value today.

Finally concerning the potential effects on the $\mathrm{CMB}$ polarization, it is well known [25] that Bianchi models give rise to polarized radiation through Thomson scattering at decoupling time. This is also the case during the reionization period and therefore a potential contribution from dark energy motion is expected also in the polarization signal at large scales. Work is in progress in this direction.

\section{ACKNOWLEDGMENTS}

We would like to thank A. de la Cruz Dombriz for useful comments. This work has been partially supported by DGICYT (Spain) under Project No. FPA 2004-02602 and No. FPA 2005-02327. The latter project has supported J. B. under the grant No. BES-2006-12059 by Ministerio de Educación y Ciencia.
[1] A. G. Riess et al., Astrophys. J. 607, 665 (2004).

[2] P. Astier et al., Astron. Astrophys. 447, 31 (2006).

[3] D. N. Spergel et al., arXiv:astro-ph/0603449; G. Hinshaw et al., arXiv:astro-ph/0603451.

[4] P. J.E. Peebles and B. Ratra, Rev. Mod. Phys. 75, 559 (2003).

[5] E. J. Copeland, M. Sami, and S. Tsujikawa, Int. J. Mod. Phys. D 15, 1753 (2006).

[6] S. Nesseris and L. Perivolaropoulos, Phys. Rev. D 70, 043531 (2004); 72, 123519 (2005); B. A. Bassett, P. S. Corasaniti, and M. Kunz, Astrophys. J. 617, L1 (2004); G. Barro Calvo and A. L. Maroto, Phys. Rev. D 74, 083519 (2006).

[7] C. Wetterich, Nucl. Phys. B302, 668 (1988); R. R. Caldwell, R. Dave, and P. J. Steinhardt, Phys. Rev. Lett. 80, 1582 (1998).

[8] G. Dvali, G. Gabadadze, and M. Porrati, Phys. Lett. B 485, 208 (2000); L. Amendola, R. Gannouji, D. Polarski, and S. Tsujikawa, Phys. Rev. D 75, 083504 (2007).

[9] R. Trotta and R. Bower, Astron. Geophys. 47, 4.20 (2006).

[10] A. Albrecht et al., arXiv:astro-ph/0609591.

[11] A. L. Maroto, J. Cosmol. Astropart. Phys. 05 (2006) 015.
[12] A. L. Maroto, Int. J. Mod. Phys. D 15, 2165 (2006); AIP Conf. Proc. 878, 240 (2006).

[13] J. D. Barrow, Phys. Rev. D 55, 7451 (1997).

[14] E. F. Bunn, P. Ferreira, and J. Silk, Phys. Rev. Lett. 77, 2883 (1996)

[15] A. de Oliveira-Costa, M. Tegmark, M. Zaldarriaga, and A. Hamilton, Phys. Rev. D 69, 063516 (2004); D. J. Schwarz, G. D. Starkman, D. Huterer, and C. J. Copi, Phys. Rev. Lett. 93, 221301 (2004).

[16] K. Land and J. Magueijo, Phys. Rev. Lett. 95, 071301 (2005).

[17] P. S. Letelier, Phys. Rev. D 22, 807 (1980); S. S. Bayin, Astrophys. J. 303, 101 (1986).

[18] V.F. Mukhanov, H. A. Feldman, and R. H. Brandenberger, Phys. Rep. 215, 203 (1992).

[19] S. Dodelson, Modern Cosmology (Academic, New York, 2003).

[20] M. Giovannini, Int. J. Mod. Phys. D 14, 363 (2005).

[21] L. Campanelli, P. Cea, and L. Tedesco, Phys. Rev. Lett. 97, 131302 (2006).

[22] S. Capozziello, A. Melchiorri, and A. Schirone, Phys. Rev. D 70, 101301 (2004); E. J. Copeland, A. R. Liddle, and D. Wands, Phys. Rev. D 57, 4686 (1998); P. G. Ferreira 
and M. Joyce, Phys. Rev. D 58, 023503 (1998).

[23] E. W. Kolb and M.S. Turner, The Early Universe (Addison-Wesley, Reading, MA, 1990).

[24] P. J. Steindhardt, L. Wang, and I. Zlatev, Phys. Rev. D 59, 123504 (1999).
[25] M. J. Rees, Astrophys. J. Lett. 153, L1 (1968); M. M. Basko and A. G. Polnarev, Mon. Not. R. Astron. Soc. 191, 207 (1980). 